\title{
The Application of Neural Networks to the Prediction of Traffic Noise
}

\author{
Shuoxian Wu and \\ Department of Architecture, South China University of Technology, Guangzhou 510640, China \\ Jiping Zhang \\ State Key Laboratory of Vibration, Shock \& Noise, Shanghai JiaoTong University, Shanghai 200030, China \\ Zhejiang Research and Design Institute of Environmental Protection, Hangzhou 310007, China
}

(Received 5 October 1999; accepted 28 April 2000)

Artificial neural networks are powerful when used in predictions. Based on the traffic noise data measured near a highway in China, an artificial neural network was formed to predict further traffic noise levels. It was proved that the predicted data are in good agreement with measured data. Therefore, the artificial neural network method provides a new method for traffic noise prediction.

\section{INTRODUCTION}

The prediction of road traffic noise is an important subject in environmental acoustics. It is valuable in environmental pollution control, management, and design planning. There are two methods typically used in predicting road traffic noise. The first is a theoretical one proposed in accordance with the physical principles of noise generation and propagation. ${ }^{1,2}$ Because practical traffic flows and peripheral topography are usually complex and may not obey the conditions of an ideal Poisson flow and a free or half-free sound field, the prediction formulas are generally expressed in some complicated forms and they are rather different from practical situations. The second one is proposed according to statistical analysis. ${ }^{3,4}$ Because detailed information of the physical mechanisms is not necessary in deriving this kind of prediction formulas, they can be applied to arbitrary traffic flows and complex peripheral circumstances. There are, however, some limitations in the use of these formulas. Obviously, both methods have their own advantages and shortcomings. There are also some hybrid prediction models ${ }^{\mathbf{5 , 6}}$ which combine features of both the above methods.

In recent years, the study of artificial neural networks (ANN) has been active. ANN have been applied extensively to pattern recognition, optimisation computation, information intelligence processing, complex control, signal processing, etc. ${ }^{7}$ However, ANN have not yet been applied to environmental acoustics. In this paper, a prediction model for road traffic noise using ANN is presented. The model is based on the traffic noise data measured near a highway in China and then it is used for prediction purposes. It is shown that the predicted data are in good agreement with measured data. This shows that the ANN technique provides a new method for traffic noise prediction.

\section{ARTIFICIAL NEURAL NETWORK}

The ANN technique is an approximate method that simulates the function of biological neural systems. It is constructed by different link models with a group of artificial neurones. Knowledge about a network is obtained through learning and training. Joints, that are able to process simple information are formed of many neurones. Each joint sends out stimulating or inhibiting signals. The information processing in the whole network is completed by mutual interactions among the joints.

At present, many kinds of $\mathrm{ANN}^{8}$ have been developed, such as the Kohonen network, Grossberg's ART network, Mutual excitation and competition network, Hopifield feedback network, Multi-layered feed-forward network, etc.. Among these, the Multi-layered feed-forward network is the most mature one and has been used extensively.

A Multi-layered feed-forward network (MFFN) includes an input layer, a hidden layer, and an output layer. The hidden layer may be just one layer or several layers. Each layer is composed of several artificial neurones. Each layer is linked with others in sequence, as shown in Fig. 1.

If the input and the output of the $j$-th neurone in the $k$-th layer are $y_{i}^{(k-1)}\left(i=1,2, \ldots, N_{k-1}\right)$ and $y_{j}^{(k)}$ respectively, the relationship between the input and the output will be:

$$
\begin{aligned}
& y_{j}^{(k)}=f_{j}^{(k)}\left[\sum_{i=1}^{N_{k-1}} w_{i j}^{(k-1)} y_{i}^{(k-1)}+\theta_{j}^{(k)}\right] ; \\
& j=1,2, \ldots, N_{k} ; \quad k=1,2, \ldots, M,
\end{aligned}
$$

where $w_{i j}^{(k-1)}$ is the link weight from the $i$-th neurone in the $(k-1)$-th layer to the $j$-th neurone in the $k$-th layer; $\theta_{j}^{(k)}$ is the threshold level of a corresponding neurone; $f_{j}^{(k)}[\cdot]$ is the transfer function of a neurone; $N_{k}$ is the number of the neurones in the $k$-th layer; and $M$ is the number of the layers.

The transfer function must be a quasi-linear one which is continuous, derivable, and monotonously incremental. It can be chosen as following Sigmoid function:

$$
f(x)=\frac{A}{1+\exp [-(B x+\theta)]},
$$

where $x$ is the input of a neurone; $\theta$ is the threshold of the neurone; $A$ and $B$ are the adjustment constants for the scale and slope respectively. 